\title{
Estado de Conservación de las especies argentinas de Kaunia (Oxylobinae, Eupatorieae, Asteraceae)
}

\author{
JESSICA N. VIERA BARRETO ${ }^{1 *}$ y GISELA SANCHO ${ }^{1}$
}

\begin{abstract}
Resumen: Las especies de Kaunia representan elementos ecológicamente importantes de las selvas subtropicales y matorrales montanos andinos. En Argentina habitan tres especies pertenecientes a este género. Dos de estas tres especies, $K$. lasiophthalma y $K$. saltensis, son componentes importantes de la Provincia Fitogeográfica de las Yungas. La tercera especie, K. rufescens, habita en la Provincia Fitogeográfica Paranaense. En el presente trabajo se brinda por primera vez un análisis del estado de conservación de las especies argentinas de Kaunia utilizando como base la metodología propuesta por la IUCN (International Union for Conservation of Nature and Natural Resources). Con la evidencia que se ha podido obtener sobre el estado general de las poblaciones de las especies de Kaunia analizadas, se obtuvo como resultado preliminar que la categoría de amenaza que mejor se adapta a su situación actual es "Menor preocupación" (LC).
\end{abstract}

Palabras clave: Argentina, Compositae, categorización, conservación, grado de amenaza.

Summary: Conservation status of the Argentinean species of Kaunia (Oxylobinae, Eupatorieae, Asteraceae). The species of Kaunia represent ecologically important elements of the Andean subtropical forests and montane scrubs. Three species of this genus live in Argentina. Two of them, K. lasiophthalma and $K$. saltensis, are important components of the Yungas Phytogeographic Province. The third species, K. rufescens, lives in the Paranaense Province. The conservation status of the Argentinean species of Kaunia is herein presented for the first time following the methodology proposed by IUCN (International Union for the Conservation of Nature and Natural Resources). Based on the evidence obtained from populations of the species of Kaunia analyzed, we preliminary assign the conservation status "Low concern" (LC) which best represents the current situation of these species in Argentina.

Key words: Argentina, Compositae, categorization, conservation, degree of threat.

\section{INTRODUCCIÓN}

El género Kaunia R. M. King \& H. Rob. incluye 11 especies distribuidas principalmente en Bolivia y extendiéndose hasta Argentina, Brasil, Paraguay, Perú y Ecuador (Viera Barreto, 2017). Las especies de este género son arbustos o pequeños árboles, con inflorescencias vistosas, numerosos capítulos y corolas usualmente blancas o violetas (Viera Barreto, 2017).

${ }^{1}$ División Plantas Vasculares, Museo de La Plata, FCNyM, UNLP

*Autor para correspondencia: jvierabarreto@fcnym.unlp. edu.ar
Las especies de Kaunia representan elementos ecológicamente importantes de las selvas subtropicales andinas y los matorrales montanos (Fig. 1A), donde habitan en ambientes diferentes especialmente en la faja arbustiva o usualmente como elementos de los bosques secundarios (Liberman Cruz \& Pedrotti, 2006). En su mayoría, las especies de este género presentan una distribución restringida y son endémicas de ciertas áreas de los bosques montanos andinos. Tal es el caso de Kaunia eucosmoides (B. L. Rob) R. M. King \& H. Rob., endémica del sur de Perú (Beltrán et al., 2006) y K. pachanoi (B. L. Rob) R. M. King \& H. Rob, endémica del sur de Ecuador. Esta última especie, inclusive, ha sido tratada en la lista roja de la UICN (International Union for the Conservation of Nature and Natural Resources) con 
Bol. Soc. Argent. Bot. 53 (1) 2018

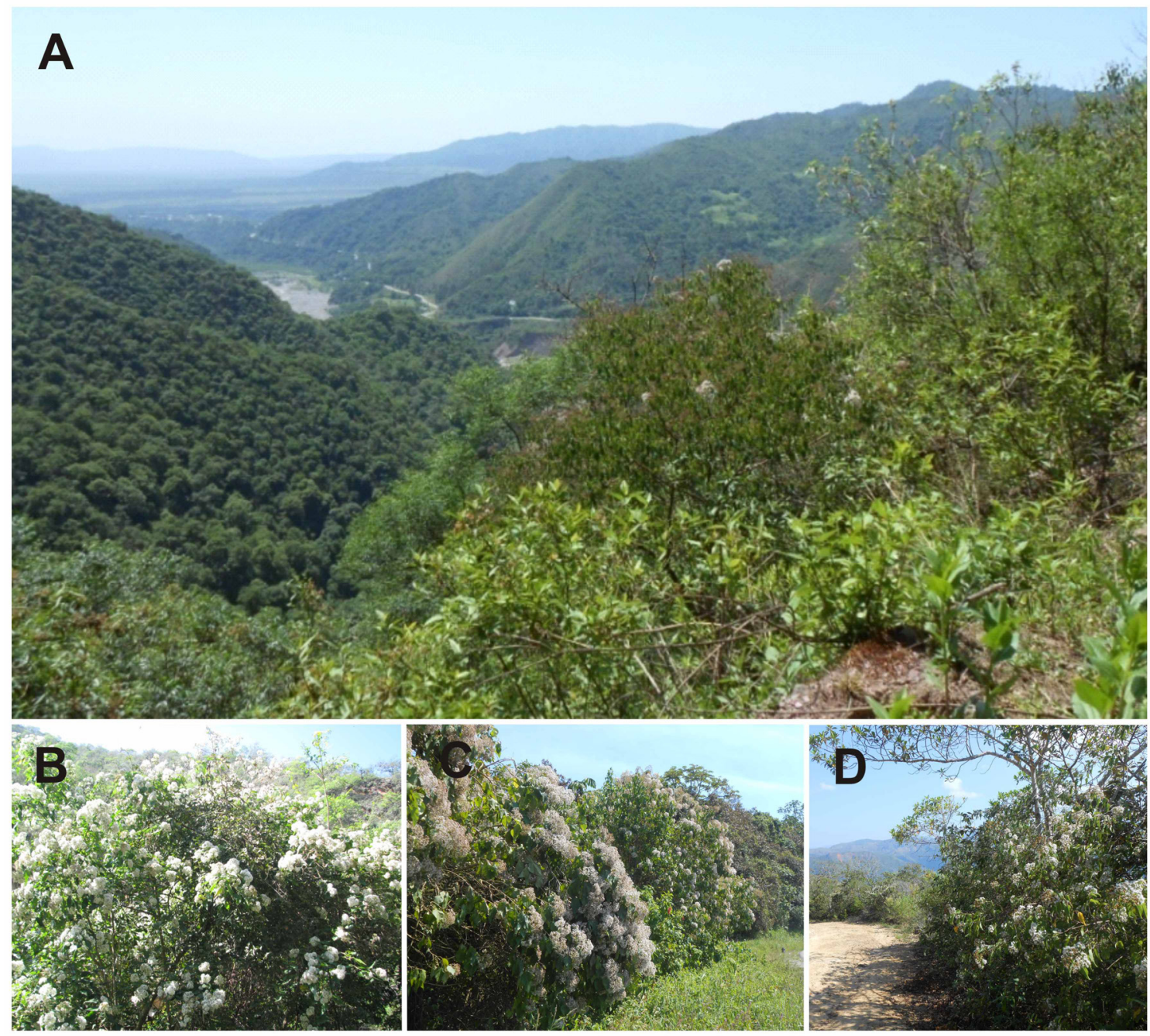

Fig. 1. A: Fotografía del ambiente montano que habitan las especies de Kaunia estudiadas, con algunos ejemplares de K. saltensis. B: Kaunia lasiophthalma. C: Kaunia rufescens. D: Kaunia saltensis.

el status de "Endangered" (en peligro de extinción) (Montúfar \& Pitman, 2003).

En la Argentina, las tres especies que pertenecen al género Kaunia son: K. lasiophthalma (Griseb.) R. M. King \& H. Rob., K. saltensis (Hieron.) R. M. King \& H. Rob. (ambas distribuidas también en Bolivia) y $K$. rufescens (P. W. Lund ex DC) R. M. King \& H. Rob. (distribuida también en Bolivia, Brasil y Paraguay) (Cabrera, 1978; Cabrera \& Klein, 1989; Cabrera et al., 1996; Jørgensen et al., 2010; Hind, 2011; Freire \& Ariza Espinar, 2014; Viera Barreto et al., 2016). Kaunia lasiophthalma (Fig. 1B) y K. saltensis (Fig. 1D) son componentes importantes de la Provincia
Fitogeográfica de las Yungas (Cabrera, 1971, 1978). Ambas especies fueron encontradas dentro de diferentes áreas protegidas del noroeste de nuestro país (Sistema de Información de Biodiversidad, www.sib.gov.ar). Kaunia lasiophthalma habita en la Provincia Biogeográfica de las Yungas principalmente entre los 900 y 1600 m s.m. (Blundo et al., 2012, Malizia et al., 2012). Esta especie también se encuentra en matorrales arbustivos (Valenzuela Celis, 1993), bosques en galería y bordes de pastizales montanos (Novara, 2009). Kaunia saltensis ha sido determinada como subdominante o acompañante en formaciones vegetales tal como los bosques de aliso 


\section{J. N. Viera Barreto y G. Sancho - Especies argentinas de Kaunia: Estado de conservación}

(Alnus jorullensis Kunth), así como también en los bosques de pino del cerro (Podocarpus parlatorei Pilg.) (Liberman Cruz \& Pedrotti, 2006; Malizia et al., 2012). El grado de amenaza de K. saltensis ha sido previamente evaluado en el marco del proyecto de Plantas Endémicas de la Argentina (www.listaplanear.org). Bajo los parámetros de este proyecto se le asignó a $K$. saltensis la categoría 4 (plantas restringidas), en base a su área de distribución y abundancia relativa de la misma. Kaunia rufescens (Fig. 1C) se encuentra citada en nuestro país para la provincia de Misiones, en el Parque Nacional Iguazú y en el Departamento de Oberá (Vega \& Dematteis, 2008). Esta especie también ha sido encontrada en las selvas del centro de Bolivia y este de Paraguay, aunque se encuentra más ampliamente distribuida en la Mata Atlántica de Brasil (Viera Barreto et al., 2016).

El sistema más ampliamente difundido para establecer prioridades de conservación es el de la Unión Internacional para la Conservación de la Naturaleza (UICN) que permite asignar una categoría de amenaza a cada especie basándose en datos sobre su demografía, distribución, persistencia del hábitat y otras contingencias previsibles. En el presente trabajo se pretende brindar por primera vez un análisis del estado de conservación de las especies argentinas de Kaunia utilizando como base la metodología propuesta por la UICN, evaluando el grado de amenaza que presentan las poblaciones de estas especies y brindando una aproximación preliminar global para la toma de decisiones relativas a la protección y manejo de sus hábitats, con énfasis en la distribución natural de estas especies en Argentina.

\section{Materiales y Métodos}

El estado de conservación de las especies argentinas de Kaunia (K. lasiophthalma, K. rufescens y $K$. saltensis) fue evaluado utilizando información de materiales de herbario (CORD, CTES, GH, LP, LPB, MO, NY, SI; Thiers, 2017), datos obtenidos de la literatura disponible, observaciones realizadas a campo durante viajes de colecta y mediante análisis cuantitativos relacionados a su distribución geográfica. Para esto se utilizaron las categorías y criterios de la lista roja de la UICN version 3.1, segunda edición (IUCN, 2012), siguiendo las "Guías para la utilización de las Categorías y Criterios de la Lista Roja de la UICN" versión 13 (IUCN, 2017).
En cuanto a la metodología propuesta por la UICN, las especies se clasifican en 9 categorías según la amenaza de extinción: EXTINCT (EX): Extinto; EXTINCT IN THE WILD (EW): Extinto en su estado Silvestre; CRITICALLY ENDANGERED (CR): En Peligro Crítico; ENDANGERED (EN): En Peligro; VULNERABLE (VU): Vulnerable; NEAR THREATENED (NT): Cerca de Amenaza; LEAST CONCERN (LC): Menor Preocupación; DATA DEFICIENT (DD): Datos Deficientes y NOT EVALUATED (NE): No Evaluado.

Según la UICN existen cinco criterios (A-E) utilizados para evaluar la pertenencia de una especie a una de las categorías de amenaza de la lista roja de la UICN: (A) reducción del tamaño poblacional (B) distribución geográfica representada como extensión de presencia y/o área de ocupación; (C) pequeño tamaño de la población y disminución; (D) población muy pequeña o restringida y $(\mathrm{E})$ análisis cuantitativo (que indique la probabilidad de extinción en estado silvestre). Para ubicar a un taxón en cualquiera de las categorías de evaluación, se necesita sólo el apoyo de uno de los criterios (A, B, C, D o E), aunque el taxón debe evaluarse bajo la mayor cantidad de criterios posibles, y sólo debe ser listado el criterio para la mayor categoría de peligro que se haya encontrado (IUCN, 2017).

Al no contar con información de naturaleza cuantitativa sobre reducción poblacional, abundancia, individuos maduros, ni genética de poblaciones, en este análisis se evaluó únicamente el criterio B (distribución geográfica representada como extensión de presencia y/o área de ocupación) para estimar el estatus de conservación de las especies. Para la evaluación mediante el criterio $\mathrm{B}$, se procedió al cálculo de la extensión de presencia (EOO) y del área de ocupación (AOO) de las tres especies en estudio.

La extensión de presencia se define como el área contenida dentro de los límites imaginarios continuos más cortos que pueden dibujarse para incluir todos los sitios conocidos, inferidos o proyectados en los que un taxón se encuentre presente, excepto los casos de vagabundeo (IUCN, 2012). La extensión de presencia puede ser medida frecuentemente por un polígono convexo mínimo (el polígono de menor superficie que contenga todos los lugares de presencia, pero que ninguno de sus ángulos internos exceda los 180 grados) (IUCN, 2012). Los umbrales críticos para EOO son $<100 \mathrm{~km}^{2}(\mathrm{CR}),<5.000 \mathrm{~km}^{2}(\mathrm{EN}) \mathrm{y}<$ $20.000 \mathrm{~km}^{2}$ (VU) (IUCN, 2012). El área de ocupación 
de un taxón se define como el área dentro de la "extensión de presencia que es ocupada por un taxón, excluyendo los casos de actividades asociadas al vagabundeo (entendiéndose por vagabundeo el caso de especies registradas una vez o esporádicamente y de las que se sabe que no son nativas del área)". La medida refleja el hecho de que un taxón por lo general no aparecerá en todo el área de su extensión de presencia, ya que ésta puede contener hábitats no ocupados o inadecuados (IUCN, 2012). Los umbrales para este parámetro son $<10 \mathrm{~km}^{2}(\mathrm{CR}),<500 \mathrm{~km}^{2}$ (EN) y $<2.000 \mathrm{~km}^{2}$ (VU). Para aplicar el criterio B, además del cálculo de la EOO y AOO, se debe tener en cuenta si las especies cumplen por lo menos dos de las tres condiciones siguientes: a) severamente fragmentada o número de localidades $<10$; b) disminución continua observada, estimada, inferida o proyectada en cualesquiera de: (i) extensión de presencia, (ii) área de ocupación, (iii) área, extensión y/o calidad del hábitat, (iv) número de localidades o subpoblaciones, (v) número de individuos maduros; c) fluctuaciones extremas en cualesquiera de: (i) extensión de presencia, (ii) área de ocupación, (iii) número de localidades o subpoblaciones, (iv) número de individuos maduros.

Para la evaluación de $K$. lasiophthalma, $K$. rufescens y $K$. saltensis primero se procedió a la georreferenciación de todos los puntos de colecta conocidos para las tres especies. Con esta información se obtuvieron los mapas de distribución para las tres especies. Luego, los polígonos convexos mínimos se generaron en el software de libre acceso QGis v. 2.8 (QGIS Development Team, 2014), utilizando la herramienta de geoproceso "Envolvente convexa", que define el polígono de menor área que contiene todos los puntos que representan a través de su localización geográfica la ocurrencia de estas especies. A partir de dichos polígonos se calculó la extensión de presencia en $\mathrm{km}^{2}$. El parámetro AOO fue calculado a una escala de $5 \times 5 \mathrm{~km}$, superponiendo una grilla de dicha resolución sobre los registros de las especies y calculando el área de cobertura de las celdas ocupadas por cada especie en el programa QGis v. 2.8 (QGIS Development Team, 2014).

Para evaluar si las especies argentinas de Kaunia cumplen con alguna de las condiciones adicionales correspondientes al criterio $\mathrm{B}$, además del cálculo de la EOO y AOO, se utilizaron datos de observaciones a campo, de etiquetas de especímenes de herbario y de la literatura disponible.

\section{Resultados}

Con los resultados de la aplicación del criterio B, teniendo en cuenta el estado general de las poblaciones de las tres especies de Kaunia analizadas, se establece preliminarmente que la categoría que mejor se adapta a su grado de amenaza actual es "Menor preocupación" (LC). En esta categoría se consideran taxones que, habiendo sido evaluados, no cumplen ninguno de los criterios que definen las categorías de En Peligro Crítico, En Peligro, Vulnerable o Casi Amenazado.

Los resultados obtenidos respecto al área de extensión de presencia de las tres especies superan el mínimo para ser consideradas como Vulnerables (< $20.000 \mathrm{~km}^{2}$ ). Para K. lasiophthalma, la extensión de presencia (EOO) obtenida fue de un valor de 243.137 $\mathrm{km}^{2}$ (Fig. 2A), para K. rufescens un valor aún mayor de $1.320 .277 \mathrm{~km}^{2}$ (Fig. 2B) y para K. saltensis 160.948 $\mathrm{km}^{2}$ (Fig. 2C).

Por otro lado, los valores obtenidos para el área de ocupación (AOO) (Fig. 3 A y B) de las tres especies corresponden a $1786 \mathrm{~km}^{2}$ para K. saltensis, $2156 \mathrm{~km}^{2}$ para K. lasiophthalma y $2102 \mathrm{~km}^{2}$ para $K$. rufescens. Como puede observarse, el valor obtenido para $K$. saltensis se ubica dentro de lo que correspondería al límite establecido para la categoría Vulnerable $(<2000$ $\mathrm{km}^{2}$ ). Sin embargo, para poder adjudicar la categoría VU a dicha especie, deben cumplirse además por lo menos dos de tres condiciones adicionales al valor de la AOO que fueron detalladas anteriormente. Además, se conocen poblaciones de ambas especies en más de 10 localidades respectivamente, por lo cual tampoco aplica este criterio para incluirlas en una categoría de peligro. Por todo lo expuesto en este análisis se concluye que las condiciones adicionales al valor de AOO y de EOO no son cumplidas por estas especies para poder adjudicarles una categoría de amenaza.

Por otro lado, luego de establecer los mapas de distribución de las especies de Kaunia que habitan las Yungas ( $K$. lasiophthalma, Fig. 4A y $K$. saltensis, Fig. 4C), se arribó a la conclusión de que en Argentina existen varias poblaciones persistentes y bien establecidas durante las últimas décadas. Esta información fue corroborada al comparar los datos de colectas de etiquetas de herbario y la observación de las mismas poblaciones en el campo (Tabla 1). En efecto, en los viajes realizados para la observación y colecta de las especies de Kaunia observamos que el 90\% de las localidades con citas previas mantenían a las 


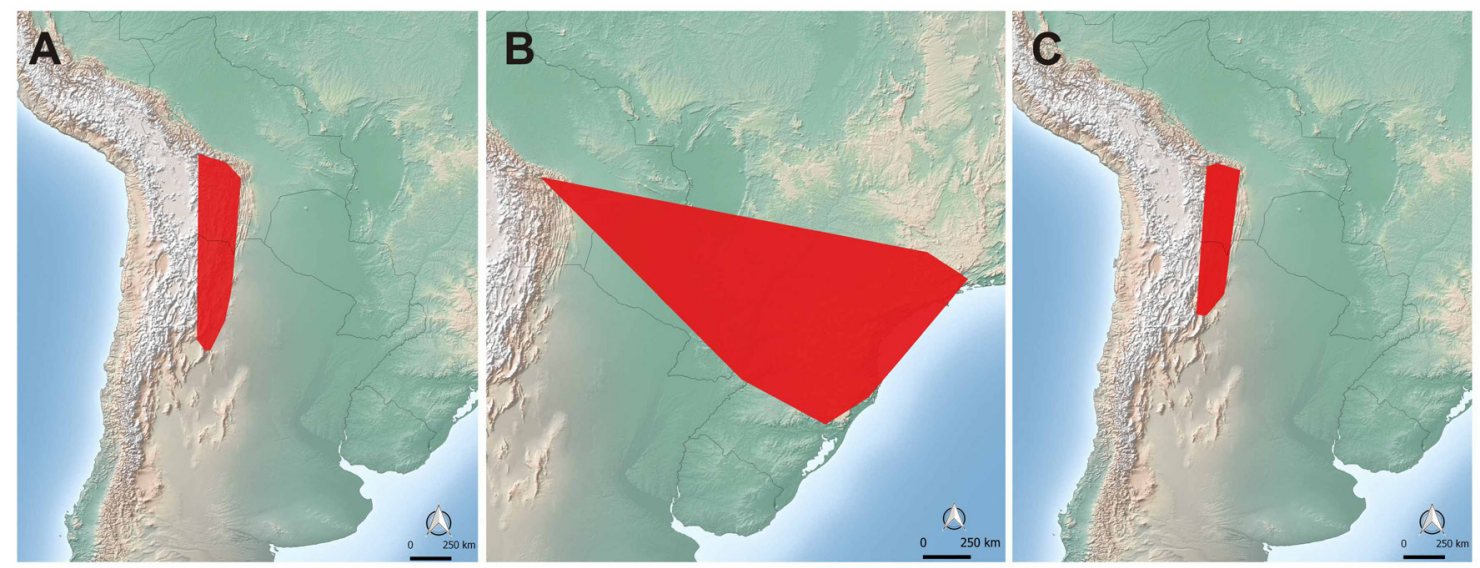

Fig. 2. A: EOO correspondiente a K. lasiophthalma. B: EOO correspondiente a K. rufescens. C: EOO correspondiente a Kaunia saltensis.

mismas poblaciones en la actualidad, por lo que no se estima o infiere una reducción poblacional considerable de estas especies. Además se ha podido observar gran cantidad de individuos de las dos especies estudiadas, sobre todo al costado de rutas y áreas disturbadas principalmente en las provincias de Salta y Jujuy, lo que se interpreta como una buena capacidad de adaptación o de crecimiento de estas especies en áreas antropizadas. No se ha documentado reducción del hábitat ni de su calidad que pudieran afectar significativamente a las poblaciones de ambas especies.
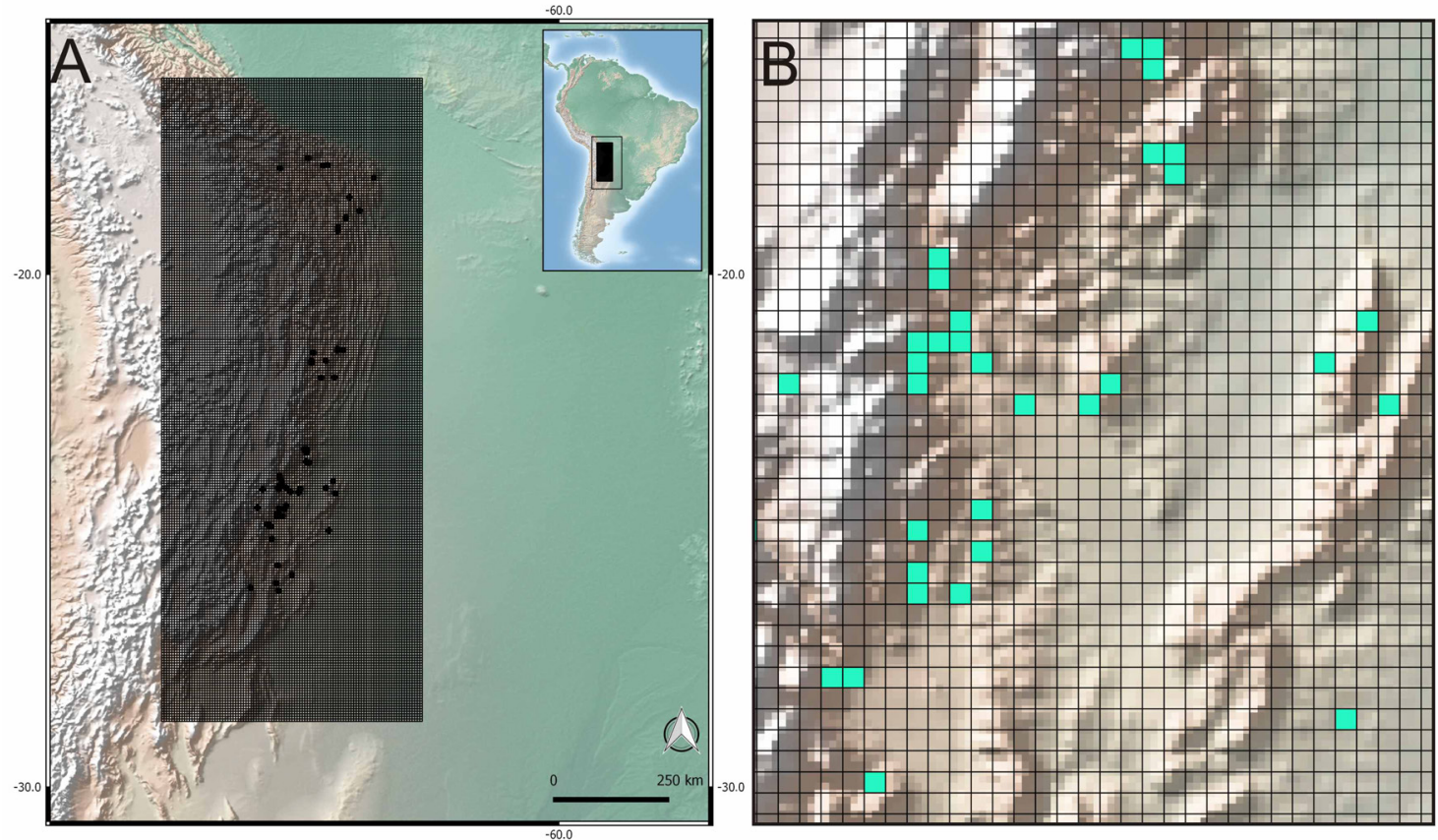

Fig. 3. A: Extensión de la grilla de $5 \times 5 \mathrm{~km}$ utilizada para evaluar el AOO de Kaunia lasiophthalma y $K$. saltensis, y la zona andina del AOO de K. rufescens. B: Detalle de la grilla donde se pueden ver resaltadas las celdas conteniendo puntos de colecta. 


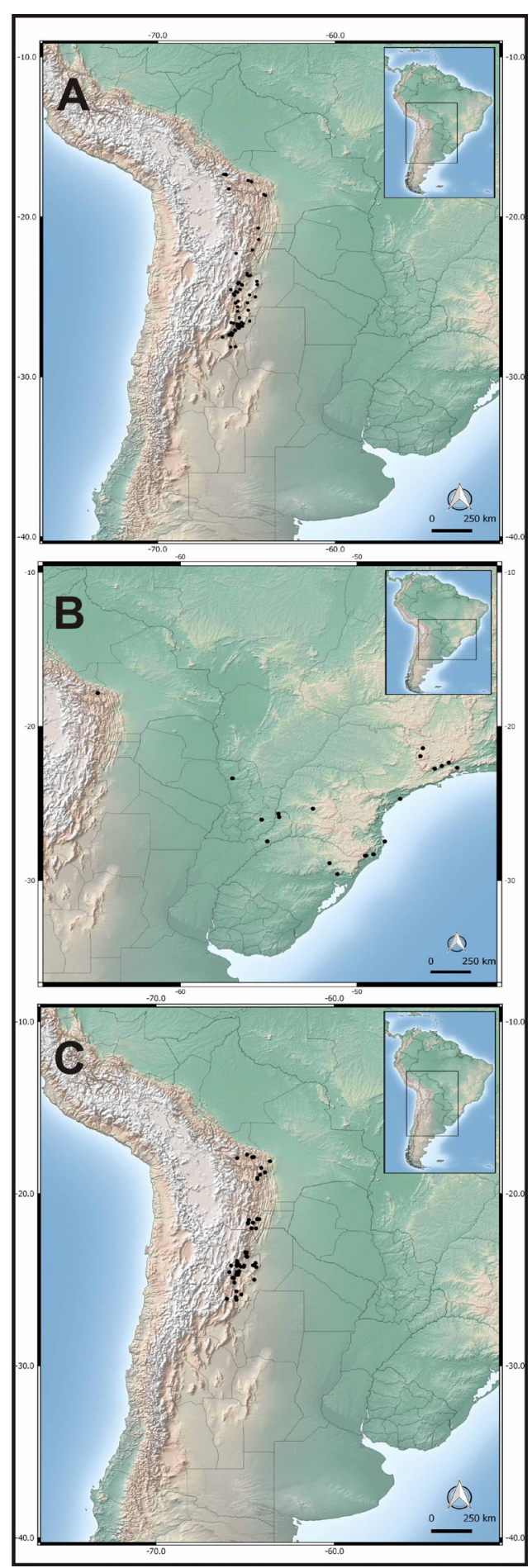

Fig. 4. A: Mapa de distribución de Kaunia lasiophthalma. B: Mapa de distribución de Kaunia rufescens. C: Mapa de distribución de Kaunia saltensis.

\section{Discusión}

Estudios realizados en las Yungas Subtropicales de Argentina (Blundo et al., 2012; Malizia et al., 2012), muestran que las especies de Kaunia que habitan en dicha región (sobre todo $K$. lasiophthalma) cobran una mayor importancia por encima de los 1600 m s.n.m. (bosque montano), aunque se superponen con pisos inferiores. $\mathrm{Si}$ bien las Yungas Subtropicales en Argentina tienen una larga historia de deforestación iniciada a fines del siglo XIX, las áreas transformadas se ubican mayoritariamente $(90 \%)$ en tierras planas por debajo del $5 \%$ de pendiente, correspondientes en su mayoría a selva pedemontana (Blundo et al., 2012), por lo que las especies de Kaunia se encuentran en un piso altitudinal con menor impacto de transformación antrópica. El bosque montano inferior (500-1700 m s.n.m., donde se encuentra gran abundancia de $K$. saltensis y $K$. lasiophthalma), puede considerarse como bosque maduro, en el sentido que no refleja claramente el efecto de disturbios antrópicos recientes a gran escala como sí puede evidenciarse en la selva pedemontana (Grau, 2005). Este tipo de bosque es el ecosistema más representado en áreas protegidas en el Noroeste Argentino (Brown, 1995). Además, las especies $K$. lasiophthalma y $K$. saltensis se encuentran bien representadas por lo menos en lo que concierne a las Áreas Protegidas Nacionales y provinciales de Argentina. Poblaciones de $K$. lasiophthalma se han citado para el Parque Nacional El Rey, Baritú y Reserva Provincial El Nogalar de los Toldos (Salta), Parque Nacional Campo de los Alisos (Tucumán) y Parque Provincial Potrero de Yala (Jujuy) (Sistema de Información de Biodiversidad, www.sib.gov.ar). En el caso de $K$. saltensis, se han detectado poblaciones en la Reserva Provincial El Nogalar de los Toldos (Salta) y en el Parque Provincial Potrero de Yala (Jujuy). La presencia de estas especies en diferentes áreas protegidas brinda un marco para su conservación.

En cuanto a los usos potenciales de estas especies que puedan representar una amenaza para las mismas, se conoce que la madera de Kaunia lasiophthalma y $K$. saltensis suele utilizarse como combustible, pero parece ser de mala calidad (Novara, 2009). No se han detectado evidencias de que las poblaciones sufrieran algún tipo de nivel de explotación real o potencial. Además, en un estudio 


\section{J. N. Viera Barreto y G. Sancho - Especies argentinas de Kaunia: Estado de conservación}

Tabla 1. Poblaciones de las tres especies argentinas de Kaunia cuya existencia se constató durante los viajes de colecta realizados en el marco de este estudio. La columna de las citas previas corresponde a los datos obtenidos de etiquetas de materiales de herbario.

\begin{tabular}{|cccc|}
\hline Especie & Localidad & $\begin{array}{c}\text { Colectas previas } \\
\text { (años) }\end{array}$ & $\begin{array}{c}\text { Última colecta } \\
\text { (año) }\end{array}$ \\
\hline K. lasiophthalma & Tucumán / Tafí del Valle & $1905-1924$ & 2013 \\
K. lasiophthalma & Salta / La Caldera & 1980 & 2013 \\
K. lasiophthalma & Jujuy / Termas de Reyes & $1928-1947-1963-1998$ & 2013 \\
K. saltensis & Jujuy / Cerro Zapla & 1980 & 2013 \\
K. saltensis & Jujuy / Termas de Reyes & $1940-1944-1945-1996-1971-2007$ & 2013 \\
K. saltensis & Salta / La Caldera & $1949-1952$ & 2013 \\
K. saltensis & Salta / Rosario de Lerma & $1929-1937-1941-1942-1958-$ & 2013 \\
K. saltensis & Bolivia / Florida & $1960-1971-1977-1986-2002$ & 2015 \\
K. saltensis & Bolivia / Camino Samaipata-Cochabamba & 1990 & 1978 \\
\hline
\end{tabular}

sobre la importancia cultural y el conocimiento sobre las especies leñosas con potencial uso agroforestal en una comunidad rural de los Andes bolivianos, $K$. saltensis mostró ser de una utilidad media y un uso activo bajo (Brandt et al., 2013).

Kaunia saltensis se encuentra dentro de la categoría 4 (plantas restringidas) según el proyecto PlanEar, que basa la asignación de categorías en parámetros diferentes a los de la UICN. Las categorías propuestas por este proyecto asigna valores del 1 al 5 a los taxones, siendo la categoría 1 la de "menor preocupación" (plantas muy abundantes en los lugares de origen y con amplia distribución geográfica en más de una de las grandes unidades fitogeográficas del país) y la categoría 5 la de "mayor preocupación" (plantas de distribución restringida, con poblaciones escasas o sobre las que se presume que puedan actuar uno o más factores de amenaza [destrucción de hábitat, sobreexplotación, invasiones biológicas, etc.]). La categoría 4, asignada a $K$. saltensis, corresponde a plantas restringidas a una sola provincia política, o con áreas reducidas compartidas por dos o más provincias políticas contiguas. Como se puede observar, la categorización de un taxón bajo este esquema depende principalmente de su distribución geográfica basada en provincias políticas. Nuestros resultados obtenidos en este estudio bajo los criterios de categorización de la UICN difieren de los obtenidos bajo el esquema de la lista Planear.
Kaunia rufescens (Fig. 4B) ha sido citada para Argentina dentro del Parque Nacional Iguazú y para el Departamento de Oberá (Provincia de Misiones) (Vega \& Dematteis, 2008). En cuanto a la primera localidad, durante la preparación de este estudio se realizó un viaje a los lugares previamente citados pero no se encontraron las poblaciones citadas para esta especie. La ausencia de poblaciones de $K$. rufescens previamente citadas para el Parque Nacional Iguazú (Vega \& Dematteis, 2008) podría estar relacionada con grandes inundaciones durante el verano de 2014, por lo que podría suponerse que los ejemplares de esta especie no han podido volver a establecerse en los mismos sitios. No hemos podido constatar el estado de la población de Oberá. Sumado a esto, es importante tener en cuenta que en la actualidad, sólo cerca del 7 $\%$ de la cobertura original de la Mata Atlántica (llamada Selva Paranaense en Argentina y Uruguay) persiste inalterada en Brasil, Argentina y Paraguay (Galindo-Leal \& Câmara, 2003). Específicamente para la Provincia de Misiones, más del $50 \%$ de la vegetación natural fue reemplazada por actividades forestales y agropecuarias, con plantaciones de pinos, araucarias, eucaliptos, yerba mate, té, tabaco y distintos tipos de ganadería (Biganzoli et al., 2004). A pesar de su enorme importancia, los bosques tropicales y subtropicales, tanto a nivel nacional como regional en Latinoamérica, sufren una permanente retracción (Cavallaro \& Fratalocchi, 2015). Estos factores podrían estar 
dificultando el establecimiento de $K$. rufescens en nuestro país. Debido al alto riesgo de pérdida de hábitat de $K$. rufescens será necesario volver a realizar observaciones de campo en los siguientes años sobre las poblaciones de Misiones para poder determinar si la especie se encuentra en peligro a nivel nacional. $K$. rufescens es utilizada ampliamente en medicina popular del sureste de Brasil, por ejemplo en Rio Grande do Sul, para tratar la tos crónica, bronquitis, gripes, para expeler piedras de los riñones, afecciones de la piel, enfermedades del útero, puntadas, dolores del cuerpo y para coagular la sangre (Inventario Florestal continuo de Rio Grande do Sul, http:// coralx.ufsm.br/ifcrs/frame.htm); sin embargo, no se ha comprobado que este consumo genere un grado de explotación importante para la especie.

Por último, no se han observado, ni se ha explicitado en la literatura ni en las observaciones de otros colectores, efectos de taxones introducidos, hibridación, patógenos, contaminantes ni parásitos, que pudieran poner en peligro la existencia de las poblaciones de alguna de las tres especies de Kaunia en estudio.

Proponemos, con toda la evidencia reunida en este estudio, asignar de forma preliminar la categoría de preocupación menor para $K$. lasiophthalma, $K$. rufescens y $K$. saltensis bajo el criterio B establecido por la UICN.

\section{Agradecimientos}

Agradecemos a los curadores de los herbarios mencionados en el texto que han hecho posible una importante parte del presente estudio. Este trabajo ha sido financiado por la Agencia Nacional de Promoción Científica y Tecnológica (ANPCyT) (BID PICT 2012-1683) y la Comisión Nacional de Investigaciones Científicas y Técnicas (CONICET) (PIP 2013/2015-0446).

\section{Biblografía}

BELTRÁN, H., A. GRANDA, B. LEÓN, A. SAGÁSTEGUI, I. SÁNCHEZ \& M. ZAPATA. 2006. Asteráceas endémicas del Perú. En: LEÓN, B., J. ROQUE, C. ULLOA, N. PITMAN. P. M. JORGENSEN \& A. CANO (eds), El libro rojo de las plantas endémicas del Perú, pp. 64-164. Revista peruana de biología Número especial 13 (2).
BIGANZOLI, F. \& M. E. MÚlgURA DE ROMERO. 2004. Inventario Florístico del Parque Provincial Teyú Cuaré y alrededores (Misiones, Argentina). Darwiniana 42: 1-24.

BLUNDO, C., L. R. MALIZIA, J. G. BLAKE \& A. D. BROWN. 2012. Tree species distribution in Andean forests: influence of regional and local factors. $J$. Trop. Ecol. 28: 83-95.

BRANDT, R., S. MATHEZ-STIEFEL, S. LACHMUTH, I. HENSEN \& S. RIST. 2013. Knowledge and valuation of Andean agroforestry species: the role of sex, and migration among members of a rural community in Bolivia. J. Ethnobiol. Ethnomed. 9: 83.

BROWN, A. D. 1995. Las selvas de montaña del nosoeste de Argentina: problemas ambientales e importancia de su conservación. En: BROWN, A. D. \& H. R. GRAU (eds.), Investigación, Conservación y Desarrollo en Selvas Subtropicales de Montaña, pp. 9-18. LIEY-Universidad Nacional de Tucumán, Tucumán.

CABRERA, A. L. 1971. Fitogeografía de la República Argentina. Bol. Soc. Argent. Bot. 14: 1-42.

CABRERA, A. L. 1978. Eupatorium. En: CABRERA, A. L. (ed.), Flora de la Provincia de Jujuy. República Argentina, pp. 106-155. Compositae- Colección Científica del INTA Buenos Aires, Colección Científica, Instituto Nacional de Tecnología Agropecuaria.

CABRERA, A. L. \& R. M. KLEIN. 1989. Compostas. 4. Tribo: Eupatorieae. Flora Ilustrada Catarinense, 1: 413-760.

CABRERA, A.L., W.C. HOLMES \& S. MCDANIEL. 1996. Compositae III, Asteroideae, Eupatorieae. Flora del Paraguay 25: 208-273.

CAVALLARO, S. \& C. FRATALOCCHI. 2015. La Planificación ecológica del territorio a través del análisis multitemporal en San Ignacio, Misiones, República Argentina. Revista Ciencias Espaciales 8: 212-230.

FREIRE, S. E. \& L. ARIZA ESPINAR. 2014. Kaunia. En: ZULOAGA F. O., M. J. BELGRANO \& A. M. ANTON (eds.), Flora Argentina. Vol. 7 (1): Dicotyledoneae, Asteraceae: Anthemidae a Gnaphalieae, pp. 363-365. Instituto de Botánica Darwinion- CONICET, San Isidro.

GALINDO-LEAL, C. Y I. G. CÂMARA 2003. The Atlantic forest of South America: biodiversity status, threats and Outlook. Island Press, Washington DC.

GRAU, H. R. 2005. Dinámica de bosques en el gradiente altitudinal de las Yungas Argentinas. En: ARTURI M. F., J. L. FRANGI \& J. F GOYA (eds), Ecología $y$ Manejo de los Bosques en Argentina, pp. 1-30. Laboratorio de Investigación de Sistemas Ecológicos y Ambientales Facultad de Ciencias Agrarias y 


\section{J. N. Viera Barreto y G. Sancho - Especies argentinas de Kaunia: Estado de conservación}

Forestales, Facultad de Ciencias Naturales y Museo, Universidad Nacional de La Plata, La Plata.

HIND, D. J. N. 2011. An annotated preliminary checklist of the Compositae of Bolivia (version 2). Disponible en: http://kew.org/science/tropamerica/ boliviacompositae.

IUCN. 2012. IUCN Red List Categories and Criteria: Version 3.1., segunda edición, IUCN Species Survival Commission, Gland, Switzerland and Cambrige, UK.

IUCN Standards and Petitions Subcommittee. 2017. Guidelines for using IUCN Red List Categories and Criteria: Version 13. Prepared by the Standards and Petitions Subcommitte. Disponible en: http://www. iucnredlist.org/documents/RedListGuidelines.pdf.

JØRGENSEN, P. M., M. NEE \& S. G. BECK. 2010. Catálogo de las plantas vasculares de Bolivia. Disponible en: http://www.tropicos.org/Project/BC.

LIBERMAN CRUZ, M. \& F. PEDROTTI. 2006. Woody formations in a mesothermic valley of Tarija Province, Bolivia. En: GAFTA, D. \& J. AKEROYD (eds.), Nature Conservation. Concepts and Practice, pp. 75-86. Springer, Berlin/Heidelberg.

MALIZIA L., S. PACHECO, C. BLUNDO \& A. D. BROWN. 2012. Caracterización altitudinal, uso y conservación de las Yungas Subtropicales de Argentina. Ecosistemas 21: 53-73.

MONTÚFAR, R. \& N. PITMAN. 2003. Kaunia pachanoi. The IUCN Red List of Threatened Species: e.T43460A10800315. Disponible en: http://dx.doi.org/10.2305/IUCN.UK.2003.RLTS. T43460A10800315.en. [Acceso: 30 Mayo 2017].

NOVARA, L. J. 2009. Tribu Eupatorieae. Flora del Valle de Lerma. (Provincia de Salta, República Argentina). Aportes Botánicos de Salta - Serie Flora 9: 1-124.
QGIS DEVELOPMENT TEAM. 2014. Quantum GIS Geographic Information System. Open Source Geospatial Foundation Project. Disponible en: http:// www.qgis.org/.

VALENZUELA CELIS, E. 1993. Compositae Giseke (Asteraceae Dumortier). En: KILLEEN, T. J., E. GARCÍA ESTIGARRIBIA \& S. G. BECK (eds.), Guía Árboles de Bolivia, pp: 222-242, Herbario Nacional de Bolivia \& Missouri Botanical Garden, Edit. Quipus srl., La Paz.

VEGA, A. J. \& M. DEMATTEIS. 2008. Eupatorium rufescens y Vernonia oligactoides (Asteraceae), nuevas citas para la Flora Argentina. Bonplandia 17: 83-89.

VIERA BARRETO, J. N., FERNANDES, A. C. y GROSSI, M. A. 2016. Género Kaunia, en Flora do Brasil 2020 online en construcción. Jardim Botânico do Rio de Janeiro. Disponible en: <http:// floradobrasil.jbrj.gov.br/reflora/floradobrasil/ FB138666>.

VIERA BARRETO, J. N. 2017. Estudios en la subtribu Oxylobinae R. M. King \& H. Rob (Asteraceae, Eupatorieae): Revisión sistemática, análisis cladístico y biogeográfico del género Kaunia R. M. King \& H. Rob. Tesis doctoral, Facultad de Ciencias Naturales y Museo, Universidad de la Plata.

THIERS, B. 2017. Index Herbariorum: A global directory of public herbaria and associated staff. New York Botanical Garden's Virtual Herbarium. Disponible en: http://sweetgum.nybg.org/ih/.

Recibido el 5 de junio de 2017, aceptado el 24 de agosto de 2017. Editor: Massimiliano Dematteis. 
